\title{
Aussicht auf eine bessere Zukunft
}

\section{Walter Fust}

\section{(2) OpenEdition}

1 Journals

Electronic version

URL: http://journals.openedition.org/sjep/517

DOI: $10.4000 /$ sjep.517

ISSN: 1663-9677

Publisher

Institut de hautes études internationales et du développement

\section{Printed version}

Date of publication: 1 novembre 2003

Number of pages: XII

ISSN: 1660-5926

\section{Electronic reference}

Walter Fust, « Aussicht auf eine bessere Zukunft », Schweizerisches Jahrbuch für Entwicklungspolitik [Online], 22-2 | 2003, Online erschienen am: 10 Juni 2010, abgerufen am 25 September 2020. URL: http://journals.openedition.org/sjep/517 ; DOI : https://doi.org/10.4000/sjep.517 


\section{Aussicht auf eine bessere Zukunft}

Die ,digitale Revolution“ gestaltet die Informationsgesellschaft des 21. Jahrhunderts. Durch die Informations- und Kommunikationstechnologien (IKT) können Informationen, Bilder und Töne gleichzeitig und in Bruchteilen von Sekunden befördert werden. Die Entfernung spielt dabei keine Rolle mehr und die Informationskanäle erlauben eine interaktive Kommunikation.

Diese Revolution betrifft alle Aspekte unseres Lebens. Sie beeinflusst unsere Art zu lernen, zu arbeiten und miteinander zu kommunizieren, wie auch die Art und Weise wie Regierungen, Privatunternehmen und die Zivilgesellschaft auf allen Ebenen interagieren.

Neue Möglichkeiten eröffnen sich jenen, welche die Informationstechnologien effizient nutzen können. Gleichzeitig bringt die „digitale Revolution“ aber auch die Gefahr mit sich, andere davon auszuschliessen, ihre Früchte zu ernten, wodurch sie die bestehenden Ungleichheiten noch erhöht. Diese Kluft zwischen jenen, die Zugang zu den IKT haben und jenen, die keinen Zugang haben, ist unter dem Namen ,digitaler Graben“ bekannt geworden.

Tatsächlich ist der „digitale Graben“ Abbild einer tiefer gehender sozialen, kulturellen und politischen Kluft. Daher spiegelt der Ausdruck ,digitale Revolution“" nur einen begrenzten Bereich der Realität wider. Ein Beispiel der sozialen Folgen der „digitalen Revolution“ ist die ,vernetzte Gesellschaft". Die einschneidende Verbesserung der technischen Vernetzungseinrichtungen hat bedeutende Auswirkungen für die sozialen, kulturellen, wirtschaftlichen und politischen Organisationen. Es gibt bereits viele Beispiele von Partnerschaften zwischen mehreren Akteuren (sog. „multi-stakeholder partnerships“) und globalen Netzwerken, welche Regierung, Zivilgesellschaft und Partner des Privatsektors auf gleichem Niveau für Interaktion, Wissensaustausch und gemeinsame Massnahmen miteinander verbinden.

Ein Partner in Bangladesch verglich die IKT mit einem Messer: es ist ein sehr wertvolles Werkzeug im Alltagsleben und im Haushalt. Jedoch kann es auch für ganz üble Dinge benutzt werden. Die IKT sind nicht „für“ oder „gegen“ Entwicklung und Armutsminderung. Sie sind ein wirksames Instrument zur Umwandlung von Informationen in Wissen und können Entwicklungsprogramme effektiver und effizienter machen. Die IKT können auch dazu eingesetzt werden, neue Ressourcen und neue Entwicklungsanstösse zu mobilisieren. Sie können die interkulturelle Kommunikation erleichtern und dadurch die Konfliktprävention stärken.

In eine weit reichende Vision und einen umfassenden Ansatz integriert, können die IKT dazu beitragen, eine bessere Zukunft für alle zu errichten.

Walter FUST

Direktor der Direktion für Entwicklung und Zusammenarbeit (DEZA) und 